\title{
Osterix transcriptional factor is involved in the metastasis of human breast cancers
}

\author{
QIANG-SHENG DAI ${ }^{1 *}$, HONG-YAN ZHOU ${ }^{2 *}$, ZHUANG-HONG WU ${ }^{3}$, JIAN-TING LONG ${ }^{1}$, \\ NAN SHAO ${ }^{3}$, TUCK-YUN CHEANG ${ }^{3}$ and SHEN-MING WANG ${ }^{3}$ \\ ${ }^{1}$ Department of Oncology; ${ }^{2}$ Neurological Intensive Care Unit; ${ }^{3}$ Department of Vascular Surgery, First Affiliated Hospital, \\ Sun Yat-Sen University, Guangzhou, Guangdong 510080, P.R. China
}

Received September 2, 2014; Accepted May 15, 2015

DOI: $10.3892 / \mathrm{ol} .2015 .3448$

\begin{abstract}
The transcriptional factor Osterix is specifically expressed in bone tissues to regulate the differentiation and maturation of osteoblasts. Recent studies have also identified the expression of Osterix in a number of cancer tissues, such as kidney and lung cancers. However, the association of Osterix with the metastasis of breast cancers has never been reported. The present study, for the first time, provides evidence supporting the involvement of Osterix in breast cancer metastasis. Western blotting was employed to investigate the expression of Osterix in a number of human breast cancer cell lines with different metastatic features. Gain-of-function and loss-of-function experiments were performed in MCF7 cells (low level of metastasis) and MDA-MB-361 cells (high level of metastasis). The expression of several metastasis-associated genes was analyzed by western blotting and quantitative polymerase chain reaction. A firefly luciferase-based reporter gene assay was conducted in order to study whether Osterix regulated the promoter activities of the MMP2 and MMP9 genes, which play critical roles in cancer metastasis. The results showed that Osterix was highly expressed in the MDA-MB-231 and MDA-MB-361 cells, but was not detectable in the MCF7 cells. The overexpression of Osterix in the MCF7 cells promoted the expression of VEGF, MMP9 and $\beta$-catenin, while downregulating the expression of E-cadherin. In addition, suppression of Osterix expression in the MDA-MB-361 cells reversed the alteration of VEGF, MMP9, $\beta$-catenin and E-cadherin expression. A reporter gene assay suggested that Osterix activated MMP2 and MMP9 promoter activity. In conclusion, Osterix is involved
\end{abstract}

Correspondence to: Dr Tuck-Yun Cheang or Dr Shen-Ming Wang, Department of Vasular Surgery, First Affiliated Hospital of Sun Yat-Sen University, 58 Zhongshan Second Road, Guangzhou, Guangdong 510080, P.R. China

E-mail: cheangtuckyun818@163.com

E-mail: wangshenming2789@163.com

*Contributed equally

Key words: Osterix, breast cancer, metastasis in the metastasis of human breast cancer and may be a target for the efficient treatment of human breast cancers.

\section{Introduction}

Osterix, also known as SP7, is a zinc finger transcription factor belonging to the specificity protein family (1). Osterix is normally expressed by osteoblasts to regulate osteoblast differentiation and maturation, and its expression is directly or indirectly regulated by various factors, including Msx 2 and BMP2 (2). Osterix can bind with the nuclear factor of activated $\mathrm{T}$ cells to form a complex, promoting osteoblastic bone formation through stimulation of the promoter activity of collagen type I $\alpha 1$ (3). In a study based on a mouse osteosarcoma model, Osterix was downregulated in mouse osteosarcoma cells and its expression was negatively associated with metastatic potency (1). Suppression of Osterix expression could inhibit osteosarcoma cell proliferation, in vitro and in vivo, reducing the metastatic potency of osteosarcoma (4). Previous studies have suggested that Osterix is a tumor suppressor gene and prevents the progression of osteosarcoma, making it a potential target for the clinical therapy of osteosarcoma (4). Osterix expression had been shown to be limited to the bone tissues; however, recent studies identified that Osterix expression was present in the metastatic stage of kidney, prostate and lung cancer, among others $(5,6)$. However, the mechanisms of transcriptional regulation and the control of cancer metastasis for this factor are poorly understood. The present study aimed to investigate whether Osterix is able to regulate genes involved in the proliferation and metastasis of breast cancer.

\section{Materials and methods}

Breast cancer cell lines. Human breast cancer cell lines, MCF7, MDA-MB-231 and MDA-MB-361, were purchased from the American Type Cell Collection (ATCC, Manassas, VA, USA), and were routinely maintained according the culture instructions provided by the ATCC. Briefly, the MCF7 cells were maintained in minimum essential medium and $10 \%$ fetal bovine serum (FBS), supplemented with $0.01 \mathrm{mg} / \mathrm{ml}$ human recombinant insulin. The MDA-MB-361 cells were cultured within Leibovitz's L-15 medium supplemented with $20 \%$ FBS, while the MDA-MB-231 cells were fed with Leibovitz's L-15 medium supplemented with 10\% FBS. 
Reagents and instruments. All culture media for the human breast cancer cell lines were purchased from Life Technologies (Carlsbad, CA, USA). The Taq polymerase and restriction enzymes for constructing the expression vectors and reporter gene vectors were obtained from New England Biolabs (Ipswich, MA, USA). The kits for electrotransformation were prepared by Lonza (Basel, Switzerland). The primary antibodies for the western blotting were products of Abcam (monoclonal rabbit anti-Osterix, 1:1,000 dilution; catalogue no. ab187158; polyclonal rabbit anti-MMP2, 1:250 dilution; catalogue no. ab110186; monoclonal rat anti- $\beta$-catenin, 1:500 dilution, catalogue no. ab22656; monoclonal mouse anti-E-cadherin, 1:500 dilution; catalogue no. ab11512; Abcam Inc., Cambridge, MA, USA). Polyclonal goat anti-mouse IRDye ${ }^{\circledR}$ 680-labeled (1:7,500 dilution; catalogue no. P/ N92668180; RD) and goat anti-rabbit IRDye 800-labeled (1:5,000 dilution; catalogue no. P/N92532211; RD) secondary antibodies were obtained from LI-COR Biosciences (Lincoln, NE, USA). The ONEGlo reagent for the detection of firefly luciferase activity was a product of Promega (catalogue no. E6120; Promega, Madison, WI, USA).

Li-COR Odyssey was used to visualize the bands on the polyvinylidene difluoride (PVDF) membrane in the western blot assay. The mRNA levels of target genes were quantified using ABI7300 (Life Technologies). The luminescence of firefly luciferase was determined with PHERAstar (BMG Labtech, Inc., Durham, NC, USA).

Generation of MCF7 subline of Osterix overexpression. The open reading frame of the human Osterix gene (GenBank accession number, AF477981.1) was synthesized by GenScript Inc., (Piscataway, NJ, USA), and subcloned into pcDNA3. $1^{+}$expression vectors. In order to generate a stable overexpression subline, the pcDNA3.1 ${ }^{+}$expression vector subcloned with Osterix was electrotransformed into MCF7 cells using Lonza kit V and Amaxa Nucleofector II. At $24 \mathrm{~h}$ post-electrotransformation, the cells were selected with G418 at a final concentration of $400 \mu \mathrm{g} / \mathrm{ml}$ for 2 weeks. Single colonies were screened by limiting dilution, and were propagated for subsequent experiments after the confirmation of Osterix expression. A subline with an empty vector was used as the control.

Generation of the MDA-MB-361 subline with Osterix-knockdown. The psiRNA-h7SK vector used for the interference of Osterix expression was purchased from Invitrogen Life Technologies. The target sequence was 5'-AAGAGGTTCACTCGTTCGGAT-3', and a universal sequence (5'-CAACAAGATGAAGAGCACCAA-3') that was not complementary to any human gene was used as a control short hairpin (sh)RNA. The shRNA vector was delivered into the MDA-MB-361 cells and stably incorporated into the MDA-MB-361 genome, as described in the generation of the Osterix-overexpression subline. The single cell-derived stable clones were obtained by limiting dilution, and were propagated for follow-up experiments.

Determination of the expression of metastasis-associated genes. The whole cell lysates from the Osterix-overexpression or Osterix-knockdown cells were prepared with RIPA
A

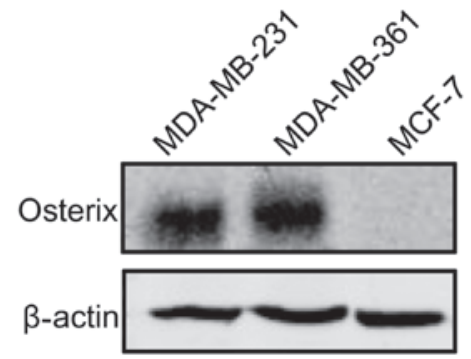

B

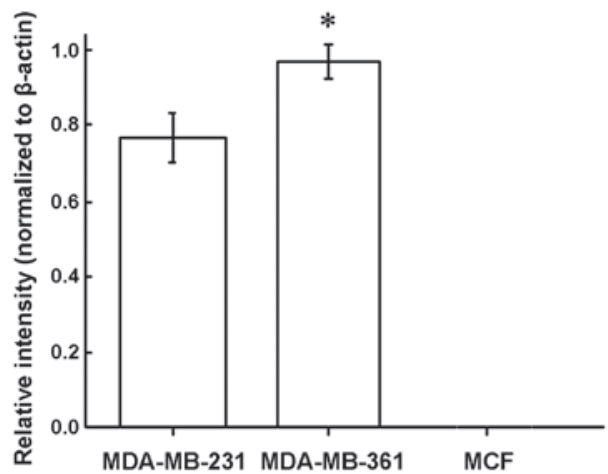

Figure 1. Osterix expression in various breast cancer cell lines. (A) The expression of Osterix in the human breast cancer MDA-MB-231, MDA-MB-361 and MCF7 cell lines was detected by western blotting. (B) Osterix was highly expressed in the MDA-MB-231 and MDA-MB-361 cell lines, while endogenous Osterix was not detectable in the MCF7 cells ${ }^{*} \mathrm{P}<0.05$ vs. MDA-MB-231 cells.

buffer for western blot analysis according to the manufacturer's instructions (Forevergen Biosciences Co., Ltd., Guangzhou, China). SDS-PAGE was conducted as described previously (7), in order to separate the proteins $(30 \mu \mathrm{g})$. For immunoblotting, proteins in the SDS gels were transferred to a PVDF membrane using electroblotting apparatus (Forevergen Biosciences, Co., Ltd.). Subsequent to being blocked with $5 \%$ bovine serum albumin in Tris-buffered saline with Tween 20, the membranes were incubated with primary antibodies against VEGF, MMP9, $\beta$-Catenin and E-cadherin at room temperature for $1.5 \mathrm{~h}$. Mouse or rabbit immunoglobulin $\mathrm{G}$ antibodies coupled with fluorescein were used as secondary antibodies. Li-COR Odyssey was used for fluorescein detection.

Assay of the MMP-2 and MMP-9 promoter activities. Firefly luciferase reporter plasmids incorporating the human MMP-2 or MMP-9 promoter were prepared using the promoter-free luciferase expression vector, pGL4.12 (Promega). The human promoter pGL4.12-MMP-2 construct extended to $-1,659 \mathrm{bp}$ relative to the transcriptional start site, and the pGL4.12-MMP-9 promoter region ranged from -992 to +304 bp relative to the transcription initiation site.

The pGL4.12-MMP-2 and pGL4.12-MMP-9 vectors were transfected into the MCF7 and MDA-MB-361 cells, respectively. The stable cell lines bearing the MMP-2 or MMP-9 promoters were established in the same way as described in the generation of the Osterix-overexpression sublines. The clonal sublines were prepared for downstream reporter gene assays after validating the basal firefly luciferase activity in the stable clones. The resultant stable clones were tentatively termed MCF7-pMMP-2, MCF7-pMM-9, MDA-MB-361-pMMP-2, and MDA-MB-361- pMMP-9. 

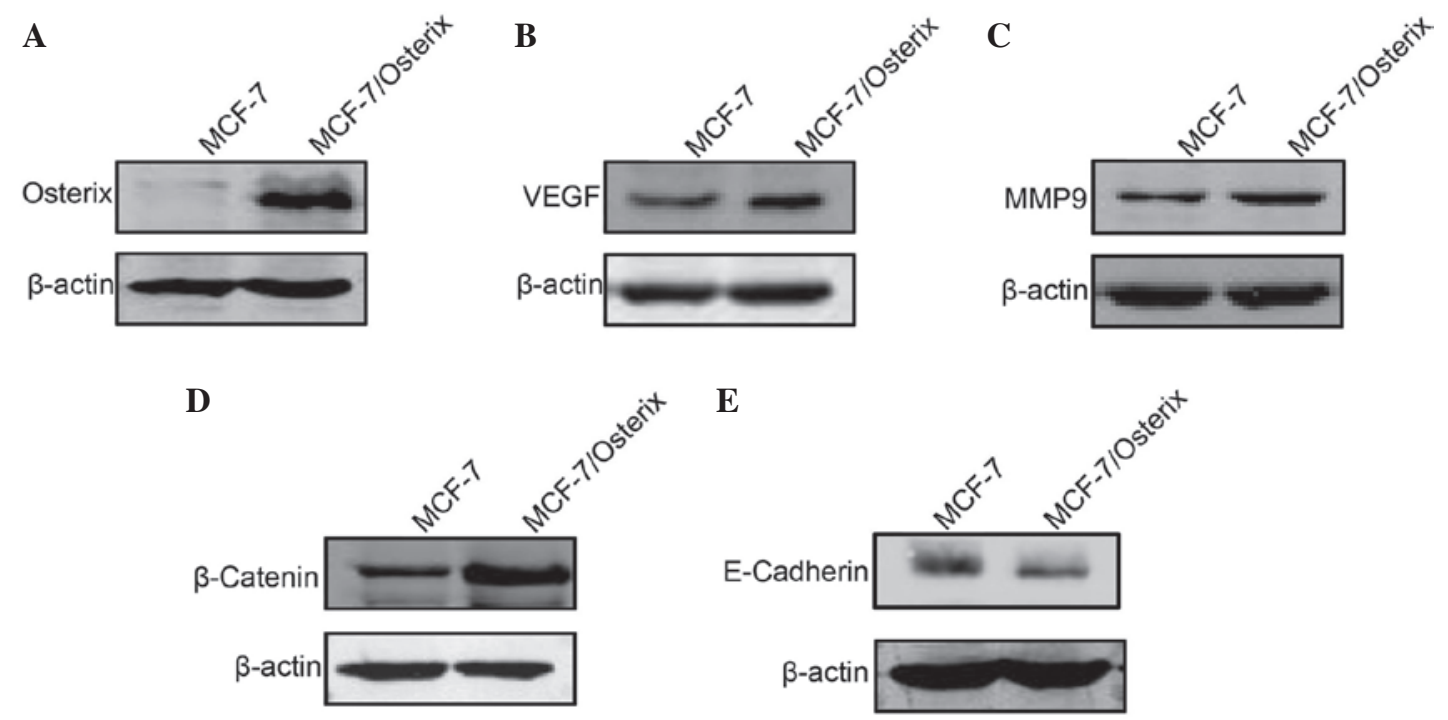

Figure 2. Overexpression of Osterix in the MCF7 cell line alters the expression of MMP9, $\beta$-catenin, E-cadherin and VEGF. Overexpression of Osterix in the MCF7 cells increased the expression levels of VEGF, MMP9 and $\beta$-catenin, while it downregulated E-cadherin expression. (A) mRNA level and Osterix-overexpression in the MCF7 cells. (B) mRNA expression level of VEGF and Osterix-overexpression in the MCF7 cells. (C) mRNA expression level of MMP9 and Osterix-overexpression in the MCF7 cells. (D) mRNA expression level of $\beta$-catenin and Osterix-overexpression in the MCF7 cells. (E) mRNA expression level of E-cadherin and Osterix-overexpression in the MCF7 cells.

The MCF7-pMMP-2 and MCF7-pMMP-9 sublines were transiently transfected with Osterix expression vectors with the Lonza electrotransformation kit, and were continuously cultured for an additional $48 \mathrm{~h}$ to allow the expression of Osterix. An equal amount of empty pcDNA. $1^{+}$vector was transfected into the cells as a control. The cells were harvested for analysis of firefly luciferase activity with PHEARstar (BMG Labtech Inc.).

The MDA-MB-361-pMMP-2 and MDA-MB-361-pMMP-9 sublines were transiently transfected with the shRNA expression vector to knock down endogenous Osterix expression. The psiRNA-h7SK vector backbone was transfected into the cells as a control. At $36 \mathrm{~h}$ post-transfection, the cells were harvested and subjected to firefly luciferase activity assay.

Statistical analysis. All statistical analyses were performed using SPSS version 17.0 software (SPSS, Inc., Chicago, IL, USA). Student's t-tests were used to compare the two groups. All experiments were repeated at least three times and data are presented as the mean \pm standard deviation. $\mathrm{P}<0.05$ was considered to indicate a statistically significant difference.

\section{Results}

Expression of Osterix in human breast cancer cell lines. Osterix has been shown to be specifically expressed in human bone tissues (1); however, until now, it was not clear whether it was also expressed in human breast cancer. In the present study, the western blotting results (Fig. 1) demonstrated that Osterix was highly expressed in the human breast cancer MDA-MB-231 and MDA-MB-361 cell lines, while the endogenous expression of Osterix was not detectable in the MCF7 cells (Fig. 1). Given that the MDA-MB-231 and MDA-MB-361 cell lines have higher metastatic potentials than the MCF7 cell line, these results suggest a role for Osterix in the metastasis of human breast cancers. Additionally, the expression of
Osterix was higher in the MDA-MB-361 cell line compared with the MDA-MB-231 cell line. Thus, MDA-MB-361 cells were used to perform subsequent Osterix knockdown experiments to investigate the expression of metastasis-associated genes in human breast cancer.

Osterix is associated with the expression of a number of metastasis-associated genes. Since the endogenous expression of Osterix was not detectable in the MCF7 cells, it was an ideal model for the investigation of the regulatory network of Osterix through the manual introduction of Osterix expression. The data showed that overexpressing Osterix altered the expression profile of a number of metastasis-related genes in the MCF7 cells. The levels of VEGF, MMP-9 and $\beta$-catenin mRNA and protein were significantly increased in the Osterix-overexpression MCF7 cells, whereas the expression of E-cadherin was suppressed (Fig. 2). Suppressing Osterix expression could reverse the alteration of the expression of VEGF, MMP-9, $\beta$-catenin and E-cadherin. Since endogenous expression of Osterix in the MDA-MB-361 cells was abundant, an shRNA expression vector containing a small interfering (si)RNA sequence against Osterix was delivered into the MDA-MB-361 cells in order to block its endogenous expression. The expression of VEGF, MMP-9, $\beta$-catenin and E-cadherin in the MDA-MB-361 cells with siRNA transfection was reversed, as expected (Fig. 3).

Osterix is involved in breast tumor metastasis through interaction with MMP-2 and MMP-9 promoters. Osterix belongs to a family of transcription factors that exert their functions through binding with the promoters of controlled genes. The overexpression or knockdown of Osterix expression could significantly enhance or reduce the expression of MMP-2 and MMP-9 (8). It has never previously been reported that Osterix could regulate the expression of MMP-2 and MMP-9 through interaction with their promoters. In the present study, the core 


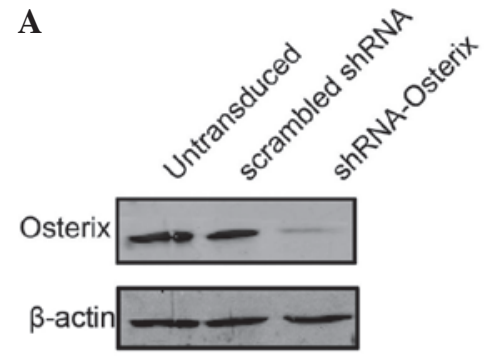

A

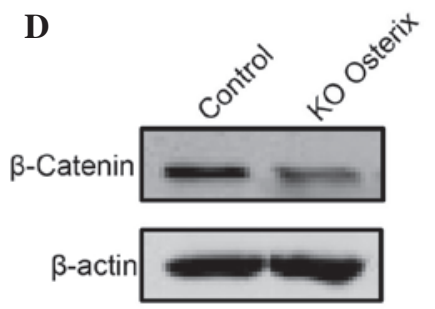

B

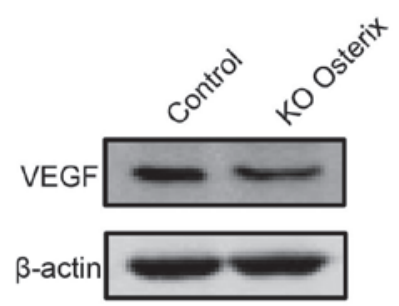

C

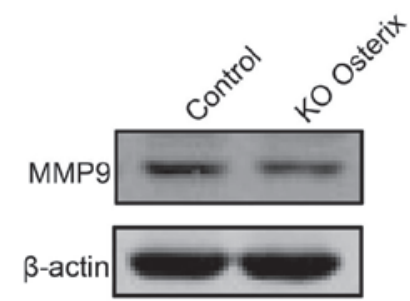

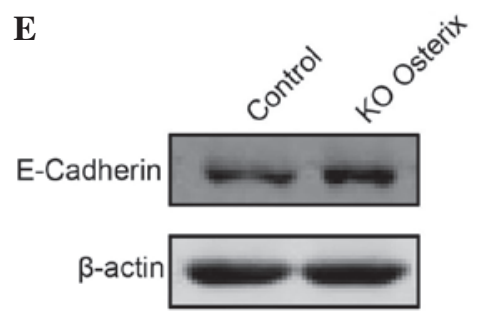

Figure 3. Knockdown of Osterix in MDA-MB-361 cells alters the expression of VEGF, MMP9, $\beta$-catenin, and E-cadherin expression. (A) mRNA expression level of Osterix and Osterix-knockdown in the MDA-MB-361 cells. (B) mRNA expression level of VEGF and Osterix-knockdown in the MDA-MB-361 cells. (C) mRNA expression level of MMP9 and Osterix-knockdown in the MDA-MB-361 cells. (D) mRNA expression level of $\beta$-catenin and Osterix-knockdown in the MDA-MB-361 cells. (E) mRNA expression level of E-cadherin and Osterix-knockdown in the MDA-MB-361 cells. shRNA, short hairpin RNA; $\mathrm{KO}$, knowckdown of Osterix.
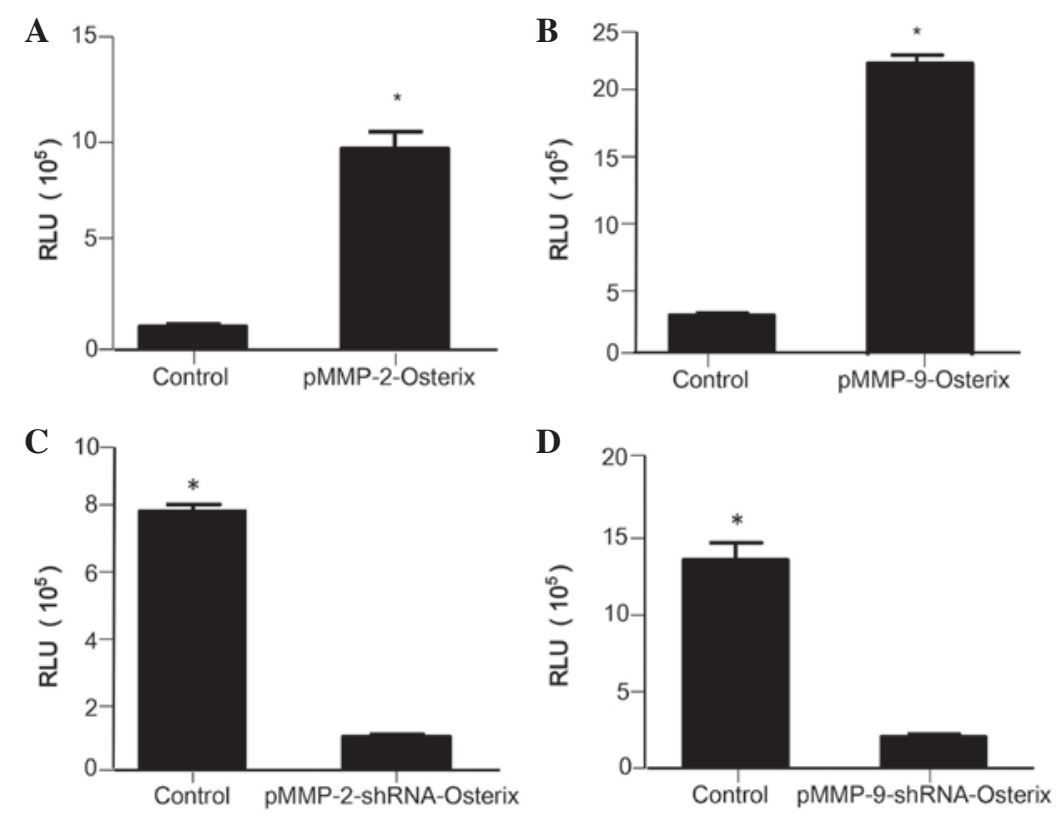

Figure 4. Analysis of the promoter activity of MMP2 and MMP9 in the MCF7 and MDA-MB-361 cells. (A) Control, MCF7 cells stably transfected with pGL4.12-MMP2 promoter and empty lentivirus; pMMP-2-Osterix, MCF7 cells stably transfected with pGL4.12-MMP2 promoter and Osterix lentivirus. (B) Control, MCF7 cells stably transfected with pGL4.12-MMP9 promoter and empty lentivirus; pMMP-2-Osterix, MCF7 cells stably transfected with pGL4.12-MMP9 promoter and Osterix lentivirus. (C) Control, MDA-MB-361 cells stably transfected with pGL4.12-MMP2 promoter and empty lentivirus; pMMP-2-shRNA-Osterix, MDA-MB-361 cells stably transfected with pGL4.12-MMP2 promoter and shRNA lentivirus against Osterix. (D) Control, MDA-MB-361 cells stably transfected with pGL4.12-MMP9 promoter and empty lentivirus; pMMP-9-shRNA-Osterix, MDA-MB-361 cells stably transfected with pGL4.12-MMP9 promoter and shRNA lentivirus against Osterix. " $\mathrm{P}<0.05$ vs. control. shRNA, small hairpin RNA.

regions of the MMP-2 and MMP-9 promoters were incorporated into the pGL4.12 vector, flanking the upstream portion of a firefly luciferase sequence. In the MCF7-pMMP-2 and MCF7-pMMP-9 stable sublines, overexpression of Osterix significantly increased the firefly luciferase activity driven by the MMP-2 or MMP-9 promoters (Fig. 4), while in the MDA-MB-361-pMMP-2 and MDA-MB-361-pMMP-9 cells, suppression of endogenous Osterix expression resulted in the inhibition of firefly luciferase activity driven by MMP-2 and MMP-9 promoters, suggesting that Osterix promoted the activities of the MMP-2 and MMP-9 promoters.

\section{Discussion}

Osterix has long been known to be a transcription factor that plays an important role in the differentiation of osteoblasts 
and bone maturation (1). However, recent studies have shown that Osterix expression is not limited to bone tissues, and expression of Osterix in metastatic kidney cancer and lung cancer has also been reported, suggesting the possible roles of Osterix in the progression of these cancers. In the present study, Osterix was found to be highly expressed in the metastatic breast cancer MDA-MB-231 and MDA-MB-361 cell lines, but undetectable in the non-metastatic breast cancer MCF7 cells, providing further evidence supporting the role of Osterix in the mediation of breast cancer metastasis. In addition, the present study identified, for the first time, the association of Osterix with a number of metastasis-associated genes, namely VEGF, MMP-9, $\beta$-catenin and E-cadherin, in human breast cancer cells. The results showed that Osterix upregulated the expression level of VEGF, MMP-9 and $\beta$-catenin, while reducing the expression of E-cadherin. A luciferase gene reporter assay demonstrated that Osterix altered the expression of MMP-2 and MMP-9 through binding with their promoters.

In the Wnt signaling pathway, $\beta$-catenin is one of the key regulators. Activation of this pathway will block proteasome-mediated degradation of $\beta$-catenin, promoting the translocation of cytoplasmic $\beta$-catenin to the nuclei, where $\beta$-catenin activates $\mathrm{T}$-cell factor/lymphoid enhancer factor transcription factors, leading to the expression of a number of oncogenes and the development of tumors $(9,10)$. In the present study, the results demonstrated that Osterix interacted with the Wnt signaling pathway through the upregulation of $\beta$-catenin and the downregulation of E-cadherin.

VEGF and its receptors play a pivotal role in angiogenesis in cancer growth (11). The binding of VEGF with VEGFR triggers multiple signaling pathways, activating the proliferation, migration and differentiation of cells, as well as increasing vascular permeability. VEGF has been reported to be associated with malignant effusions $(12,13)$. VEGF overexpression has been found to be associated with the progression and poor prognosis of several tumors, including human breast cancer (14). In the present study, Osterix upregulated VEGF expression, suggesting that Osterix promoted breast cancer progression through this pathway.

MMPs, particularly MMP-2 and MMP-9, are known to be involved in angiogenesis, tumor growth and metastasis due to their ability to degrade the extracellular matrix, leading to the endothelial cell migration (15-18). MMP-2 and MMP-9 secretion is elevated in several types of human cancers and their elevated expression has been associated with the poor prognosis of cancer patients (19-21). The present study employed a firefly luciferase-based reporter assay to analyze the interaction of Osterix with the MMP2 and MMP9 promoters. The results showed that Osterix overexpression significantly enhanced MMP2 and MMP9 promoter activities.

Taken together, these results indicate that Osterix plays a pivotal role in the metastasis of human breast cancer and may be a novel target for the efficient treatment of human breast cancers.

\section{Acknowledgements}

This study was supported by grants from the Science and Technology Planning Project of Guangdong Province (no. 2012B031800032) and the National Natural Science Foundation of China (no. 81372821).

\section{References}

1. Nakashima K, Zhou X, Kunkel G, Zhang Z, Deng JM, Behringer RR and de Crombrugghe $B$ : The novel zinc finger-containing transcription factor osterix is required for osteoblast differentiation and bone formation. Cell 108: 17-29, 2002.

2. Matsubara T, Kida K, Yamaguchi A, Hata K, Ichida F, Meguro H, Aburatani H, Nishimura R and Yoneda T: BMP2 regulates Osterix through Msx2 and Runx2 during osteoblast differentiation. J Biol Chem 283: 29119-29125, 2008.

3. Koga T, Matsui Y, Asagiri M, Kodama T, de Crombrugghe B, Nakashima K and Takayanagi H: NFAT and Osterix cooperatively regulate bone formation. Nat Med 11: 880-885, 2005.

4. Cao Y, Zhou Z, de Crombrugghe B, Nakashima K, Guan H, Duan X, Jia SF and Kleinerman ES: Osterix, a transcription factor for osteoblast differentiation, mediates antitumor activity in murine osteosarcoma. Cancer Res 65: 1124-1128, 2005.

5. Logothetis CJ and Lin SH: Osteoblasts in prostate cancer metastasis to bone. Nat Rev Cancer 5: 21-28, 2005.

6. Steeg PS: Tumor metastasis: Mechanistic insights and clinical challenges. Nat Med 12: 895-904,2006.

7. Schägger $\mathrm{H}$ and von Jagow $\mathrm{G}$ : Tricine-sodium dodecyl sulfate-polyacrylamide gel electrophoresis for the separation of proteins in the range from 1 to $100 \mathrm{kDa}$. Anal Biochem 166: 368-379, 1987.

8. Yang D, Guo Q, Guo Y, Peng Y and Ma C: Expression of osterix in metastatic breast cancer cells and its effects on MMP-2, MMP-9 and VEGF promoter activities. Suzhou University Journal of Medical Science 3: 6, 2010

9. Clevers H: Wnt/beta-catenin signaling in development and disease. Cell 127: 469-480, 2006.

10. Grigoryan T, Wend P, Klaus A and Birchmeier W: Deciphering the function of canonical Wnt signals in development and disease: Conditional loss- and gain-of-function mutations of beta-catenin in mice. Genes Dev 22: 2308-2341, 2008.

11. Ferrara N, Gerber H and LeCouter J: The biology of VEGF and its receptors. Nat Med 9: 669-676, 2003.

12. Zebrowski BK, Yano S, Liu W, Shaheen RM, Hicklin DJ, Putnam JB Jr and Ellis LM: Vascular endothelial growth factor levels and induction of permeability in malignant pleural effusions. Clin Cancer Res 5: 3364-3368, 1999.

13. Zebrowski BK, Liu W, Ramirez K, Akagi Y, Mills GB and Ellis LM: Markedly elevated levels of vascular endothelial growth factor in malignant ascites. Ann Surg Oncol 6: 373-378, 1999.

14. Berns EM, Klijn JG, Look MP, Grebenchtchikov N, Vossen R, Peters H, Geurts-Moespot A, Portengen H, van Staveren IL, Meijer-van Gelder ME, et al: Combined vascular endothelial growth factor and TP53 status predicts poor response to tamoxifen therapy in estrogen receptor-positive advanced breast cancer. Clin Cancer Res 9: 1253-1258, 2003.

15. Coussens LM, Fingleton B and Matrisian LM: Matrix metalloproteinase inhibitors and cancer: Trials and tribulations. Science 295: 2387-2392, 2002.

16. Curran S and Murray GI: Matrix metalloproteinases: Molecular aspects of their roles in tumour invasion and metastasis. Eur $\mathrm{J}$ Cancer 36: 1621-1630, 2000.

17. Egeblad M and Werb Z: New functions for the matrix metalloproteinases in cancer progression. Nat Rev Cancer 2: 161-174, 2002.

18. Hoekstra R, Eskens FA and Verweij J: Matrix metalloproteinase inhibitors: Current developments and future perspectives. Oncologist 6: 415-427, 2001

19. Zheng H, Takahashi H, Murai Y, Cui Z, Nomoto K, Niwa H, Tsuneyama K and Takano Y: Expressions of MMP-2, MMP-9 and VEGF are closely linked to growth, invasion, metastasis and angiogenesis of gastric carcinoma. Anticancer Res 26: 3579-3583, 2006.

20. Sier CF, Kubben FJ, Ganesh S, Heerding MM, Griffioen G, Hanemaaijer R, van Krieken JH, Lamers CB and Verspaget HW: Tissue levels of matrix metalloproteinases MMP-2 and MMP-9 are related to the overall survival of patients with gastric carcinoma. Brit J Cancer 74: 413, 1996.

21. Liabakk N, Talbot I, Smith RA, Wilkinson K and Balkwill F: Matrix metalloprotease 2 (MMP-2) and matrix metalloprotease 9 (MMP-9) type IV collagenases in colorectal cancer. Cancer Res 56: 190-196, 1996. 